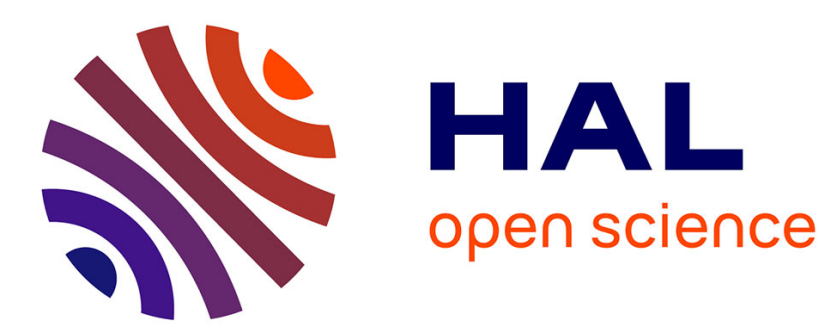

\title{
Sharing a resource with randomly arriving foragers
}

Pierre Bernhard, Frédéric Marie Hamelin

\section{To cite this version:}

Pierre Bernhard, Frédéric Marie Hamelin. Sharing a resource with randomly arriving foragers. Mathematical Biosciences, 2016, 273 (91), pp.91-101. 10.1016/j.mbs.2016.01.004 . hal-01294950

\section{HAL Id: hal-01294950 \\ https://hal.inria.fr/hal-01294950}

Submitted on 31 Mar 2016

HAL is a multi-disciplinary open access archive for the deposit and dissemination of scientific research documents, whether they are published or not. The documents may come from teaching and research institutions in France or abroad, or from public or private research centers.
L'archive ouverte pluridisciplinaire HAL, est destinée au dépôt et à la diffusion de documents scientifiques de niveau recherche, publiés ou non, émanant des établissements d'enseignement et de recherche français ou étrangers, des laboratoires publics ou privés. 


\title{
Sharing a resource with randomly arriving foragers
}

\author{
Pierre Bernhard ${ }^{\mathrm{a}, *}$, Frédéric Hamelin ${ }^{\mathrm{b}}$ \\ a BIOCORE team, INRIA-Sophia Antipolis Méditerranée, B.P. 93, F-06902 Sophia Antipolis Cedex, France \\ ${ }^{\mathrm{b}}$ AGROCAMPUS OUEST, UMR 1349 IGEPP, F-35042 Rennes Cedex, France
}

\section{A R T I C L E I N F O}

\section{Article history:}

Received 4 May 2015

Revised 25 November 2015

Accepted 11 January 2016

Available online 19 January 2016

\section{Keywords:}

Foraging

Functional response

Random population

Poisson process

\begin{abstract}
A B S T R A C T
We consider a problem of foraging where identical foragers, or predators, arrive as a stochastic Poisson process on the same patch of resource. We provide effective formulas for the expected resource intake of any of the agents, as a function of its rank, given their common functional response. We give a general theory, both in finite and infinite horizon, and show two examples of applications to harvesting a common under different assumptions about the resource dynamics and the functional response, and an example of application on a model that fits, among others, a problem of evolution of fungal plant parasites.
\end{abstract}

(c) 2016 Elsevier Inc. All rights reserved.

\section{Introduction}

The theory of foraging and predation has generally started with the investigation of the behavior of a lone forager $[3,15]$ or of an infinite population of identical foragers, investigating the effect of direct competition $[18,19,28]$ or their spatial distribution $[7,16,17]$. Then, authors investigated fixed finite groups of foragers in the concept of "group foraging" $[4,8,9]$.

This article belongs to a fourth family where one considers foragers arriving as a random process. Therefore, there are a finite number of them at each time instant, but this number is varying with time (increasing), and a priori unbounded. We use a Poisson process as a model of random arrivals. Poisson processes have been commonly used in ecology as a model of encounters, either of a resource by individual foragers, or of other individuals $[1,26]$. However, our emphasis is on foragers (or predators) arriving on a given resource. There do not seem to be many examples of such setups in the existing literature. Some can be found, e.g. in [1012], and also [29] (mainly devoted to wireless communications, but with motivations also in ecology).

In [11], the authors consider the effect of the possibility of arrival of a single other player at a random time on the optimal diet selection of a forager. In [10,12], the authors consider an a priori unbounded series of arrivals of identical foragers, focusing on the patch leaving strategy. In these articles, the intake rate as a function of the number of foragers - or functional response- is

\footnotetext{
* Corresponding author.

E-mail addresses: Pierre.Bernhard@inria.fr (P. Bernhard), fhamelin@agrocampusouest.fr (F. Hamelin).
}

within a given family, depending on the density of resource left on the patch and on the number of foragers (and, in [12] on a scalar parameter summarizing the level of interference between the foragers). And because the focus is on patch leaving strategies, one only has to compare the current intake rate with an expected rate in the environment, averaged over the equiprobable ranks of arrival on future patches.

In the current article, we also consider an a priori unbounded series of random arrivals of identical foragers, but we focus on the expected harvest of each forager, as a function of its rank and arrival time. Our aim is to give practical means of computing them, either through closed formulas or through efficient numerical algorithms. These expressions may later be used in foraging theory, e.g. in the investigation of patch leaving strategies or of joining strategies [25].

In Section 2, we first propose a rather general theory where the intake rate is an arbitrary function of the state of the system. All foragers being considered identical, this state is completely described by the past sequence of arrivals and current time.

In Section 3, we offer three particular cases with specific resource depletion rates and functional responses, all in the case of "scramble competition" (see [10]). But there is no a priori obstruction to dealing also with interference. The limitation, as we shall see, is in the complexity of the dynamic equation we can deal with.

We only consider the case of a Poisson process of arrivals, making the harvesting process of any player a Piecewise Deterministic Markov Process (PDMP). Such processes have been investigated in the engineering literature, since [27] and [24] at least. As far as we know, the term PDMP (and even PDMDP for Piecewise 
Deterministic Markov Decision Process, but we have no decision here) was first introduced in [5]. Their control, the decision part, was further investigated, in e.g. [6,31] and a wealth of literature. Later articles such as $[2,13]$ have concentrated on asymptotic properties of their optimal trajectories, and applications in manufacturing systems.

These articles (except [5] who proposes general tools for PDMP parallel to those available for diffusion processes) focus on existence and characterization of optimal control strategies. When they give means of calculating the resulting expected payoff, it is through a large (here infinite) set of coupled Hamilton-Jacobi (hyperbolic) partial differential equations. Here, we want to focus our attention on the problem of evaluating this payoff when the intake rates, the equivalent of strategy profiles of the control and games literature, for each number of players present on the common, are given; typically a known functional response. We take advantage of the very simple structure of the underlying jump process (discussed below), and of the continuous dynamics we have, to obtain closed form, or at least numerically efficient, expressions for the expected payoff, which we call Value for brevity.

\section{General theory}

\subsection{Notation}

Data: $t_{1}, T, \lambda,\left\{L_{m}(\cdot, \cdot), m \in \mathbb{N}\right\}$.

Result sought $V_{n}(\cdot), n \in \mathbb{N}$.

$t_{1} \in \mathbb{R} \quad$ Beginning of the first forager's activity.

$T \in\left(t_{1}, \infty\right] \quad$ Time horizon, either finite or infinite.

$t \in\left[t_{1}, T\right] \quad$ Current time.

$m(t) \in \mathbb{N} \quad$ Number of foragers present at time $t$.

$t_{m} \quad$ Arrival time of the $m$ th forager. (A Poisson process.)

$\tau_{m} \quad$ Sequence $\left(t_{2}, t_{3}, \ldots t_{m}\right)$ of past arrival times.

$\mathcal{T}_{m}(t) \subset \mathbb{R}^{m-1} \quad$ Set of consistent $\tau_{m(t)}:\left\{\left(\tau_{m} \mid t_{1}<t_{2} \cdots<t_{m} \leq t\right\}\right.$.

$\lambda \in \mathbb{R}_{+} \quad$ Intensity of the Poisson process of arrivals.

$\delta \in \mathbb{R}_{+} \quad$ Actualization factor (intensity of the random death process).

$L_{m}\left(\tau_{m}, t\right) \in \mathbb{R}_{+}$Intake rate of all foragers when they are $m$ on the common.

$M_{m}(t) \in \mathbb{R}_{+} \quad$ Sum of all possible $L_{m(t)}$, for all possible $\tau_{m} \in$ $\mathcal{T}_{m}(t)$.

$J_{m}\left(\tau_{m}\right) \quad$ Reward of forager with arrival rank $m$, given the sequence $\tau_{m}$ of past arrival times. (A random variable.)

$V_{1} \in \mathbb{R}_{+} \quad$ First forager's expected reward.

$V_{m}\left(\tau_{m}\right) \in \mathbb{R}_{+} \quad$ Expected reward of the forager of rank $m$.

$J_{m}^{(n)}\left(\tau_{m}\right) \quad$ Reward of player $m$ if the total number of arriving foragers is bounded by $n$. (Random variable)

$V_{m}^{(n)}\left(\tau_{m}\right) \in \mathbb{R}_{+} \quad$ Expectation of $J_{m}^{(n)}\left(\tau_{m}\right)$.

\subsection{Statement of the problem}

We aim to compute the expected harvest of foragers arriving at random, as a Poisson process, on a resource that they somehow have to share with the other foragers, both those already arrived and those that could possibly arrive later. At this stage, we want to let the process of resource depletion and foraging efficiency be arbitrary. We shall specify them in the examples of Section 3.

\subsubsection{Basic notation}

We assume that there is a single player at initial time $t_{1}$. Whether $t_{1}$ is fixed or random will be discussed shortly. At this stage, we let it be a parameter of the problem considered. Then identical players arrive as a Poisson process of intensity $\lambda$, player number $m$ arriving at time $t_{m}$. The state of the system, (if $t_{1}$ is fixed) is entirely characterized by the current time $t$, the current number of foragers arrived $m(t)$, and the past sequence of arrival times that we call $\tau_{m(t)}$ :

$\forall m \geq 2, \quad \tau_{m}:=\left(t_{2}, t_{3}, \ldots, t_{m}\right)$,

a random vector. The intake rate of any forager at time $t$ is therefore a function $L_{m(t)}\left(\tau_{m(t)}, t\right)$.

Let the horizon be $T$, finite or infinite. We may just write the payoff of the first player as

$J_{1}\left(t_{1}\right)=\int_{t_{1}}^{T} \mathrm{e}^{-\delta\left(t-t_{1}\right)} L_{m(t)}\left(t_{2}, \ldots, t_{m(t)}, t\right) \mathrm{d} t$.

(We will often omit the index 1 and the argument $t_{1}$ of $J_{1}$ or $V_{1}$.) We shall also be interested in the payoff of the $n$th player arrived:

$J_{n}\left(\tau_{n}\right)=\int_{t_{n}}^{T} \mathrm{e}^{-\delta\left(t-t_{n}\right)} L_{m(t)}\left(\tau_{m(t)}, t\right) \mathrm{d} t$.

(We shall often, in such formulas as above, write $m$ for $m(t)$ when no ambiguity results.) The exponential actualization $\exp (-\delta t)$ will be discussed shortly. We always assume $\delta \geq 0$. In the finite horizon problem, it may, at will, be set to $\delta=0$.

\subsubsection{Initial time $t_{1}$}

In all our examples, the functions $L_{m}\left(\tau_{m}, t\right)$ only depend on time through differences $t-t_{1}$, or $t-t_{m}, t_{m}-t_{m-1}, \ldots t_{2}-t_{1}$. They are shift invariant. We believe that this will be the case of most applications one would think of. In such cases, the results are independent of $t_{1}$. Therefore, there is no point in making it random.

If, to the contrary, the time of the day, say, or the time of the year, enters into the intake rate, then it makes sense to consider $t_{1}$ as a random variable. One should then specify its law, may be exponential with the same coefficient $\lambda$, making it the first event of the Poisson process. In this case, our formulas actually depend on $t_{1}$, and the various payoff $V_{n}$ should be taken as the expectations of these formulas.

One notationally un-natural way of achieving this is to keep the same formulas as below (in the finite horizon case), let $t_{1}=0$, and decide that, for all $m \geq 2, t_{m}$ is the arrival time of the forager number $m-1$. A more natural way is to shift all indices by one, i.e. keep the same formulas, again with $t_{1}=0$, and decide that $\tau_{m}:=\left(t_{1}, t_{2}, \ldots, t_{m}\right)$, and $\mathcal{T}_{m}(t)=\left\{\tau_{m} \mid 0<t_{1}<\cdots<t_{m} \leq t\right\}$.

\subsubsection{Horizon $T$}

The simplicity of the underlying Markov process in our Markov Piecewise Deterministic Process stems from the fact that we do not let foragers leave the resource before $T$ once they have joined. The main reason for that is based upon standard results of foraging theory that predict that all foragers should leave simultaneously, when their common intake rate drops below a given threshold. (See $[3,10,12]$.)

When considering the infinite horizon case, we shall systematically assume that the system is shift invariant, and, for simplicity, let $t_{1}=0$. A significant achievement of its investigation is in giving the conditions under which the criterion converges, i.e. how it behaves for a very long horizon. Central in that question is the exponential actualization factor. As is well known, it accounts for the case where the horizon is not actually infinite, but where termination will happen at an unknown time, a random horizon with an exponential law of coefficient $\delta$. It has the nice feature to let a bounded revenue stream give a bounded pay-off. Without this discount factor, the integral cost might easily be undefined. In that respect, we just offer the following remark:

Proposition 1. If there exists a sequence of positive numbers $\left\{\ell_{m}\right\}$ such that the infinite series $\Sigma_{m} \ell_{m}$ converges, and the sequence of functions $\left\{L_{m}(\cdot)\right\}$ satisfies a growth condition

$\forall m \in \mathbb{N}, \quad \forall$ sequences $\left(t_{2}, t_{3}, \ldots, t_{m}, t\right), \quad\left|L_{m}\left(t_{2} \ldots, t_{m}, t\right)\right| \leq \ell_{m}$, 
then the infinite horizon integral converges even with $\delta=0$.

As a matter of fact, we then have

$\left|J_{1}\right| \leq \int_{0}^{\infty}\left|L_{m(t)}\right| \mathrm{d} t \leq \int_{0}^{\infty} \ell_{m(t)} \mathrm{d} t=\sum_{m=1}^{\infty}\left(t_{m+1}-t_{m}\right) \ell_{m}$,

and hence

$\mathbb{E} J_{1} \leq \frac{1}{\lambda} \sum_{m=1}^{\infty} \ell_{m}$,

ensuring the convergence of the integral. (Notice however that this is not satisfied by the first two examples below, and not an issue for the third.)

\subsubsection{Further notation}

We will need the following notation:

$\forall m \geq 2, \quad \mathcal{T}_{m}(t)=\left\{\tau_{m} \in \mathbb{R}^{m-1} \mid t_{1}<t_{2}<\cdots<t_{m} \leq t\right\}$,

(a better notation would be $\mathcal{T}_{m}\left(t_{1}, t\right)$, but we omit $t_{1}$ for simplicity) and

$M_{1}(t)=L_{1}(t), \quad \forall m \geq 2, \quad M_{m}(t)=\int_{\mathcal{T}_{m}(t)} L_{m}\left(\tau_{m}, t\right) \mathrm{d} \tau_{m}$.

More explicitly, we can write, for example

$$
\begin{aligned}
M_{3}(t) & =\int_{t_{1}}^{T} \mathrm{~d} t_{2} \int_{t_{2}}^{T} \mathrm{~d} t_{3} \int_{t_{3}}^{T} L_{3}\left(t_{2}, t_{3}, t\right) \mathrm{d} t \\
& =\int_{t_{1}}^{T} \mathrm{~d} t \int_{t_{1}}^{t} \mathrm{~d} t_{3} \int_{t_{1}}^{t_{3}} L\left(t_{2}, t_{3}, t\right) \mathrm{d} t_{2} .
\end{aligned}
$$

These $M_{m}(t)$ are deterministic functions, which will be seen to sufficiently summarize the $L_{m}\left(\tau_{m}, t\right)$, a huge simplification in terms of volume of data. The explanation of their appearance is in the fact that once $t$ and $m(t)$ are given, all sequences $\tau_{m} \in \mathcal{T}_{m}(t)$ are equiprobable. That is why only their sum comes into play. It will be weighted by the probability that that particular $m(t)$ happens.

To give a precise meaning to this notation, we make the following assumption, where $\overline{\mathcal{T}_{m}(t)}$ stands for the closure of the set $\mathcal{T}_{m}(t)$ :

Assumption 1. Let $\mathcal{D}_{m}$ be the domain $\left\{\left(t, \tau_{m}\right) \in \mathbb{R} \times \mathbb{R}^{m-1} \mid \tau_{m} \in\right.$ $\left.\overline{\mathcal{T}_{m}(t)}\right\} . \forall m \in \mathbb{N} \backslash\{1\}$, the functions $L_{m}(\cdot, \cdot)$ are continuous from $\mathcal{D}_{m}$ to $\mathbb{R}$.

As a consequence, the fact that $M_{m}$ be defined as an integral over a non-closed domain is harmless. For integration purposes, we may take the closure of $\mathcal{T}_{m}$. This also implies that each of the $L_{m}(\cdot, t)$ is bounded. Concerning the bounds, we give two definitions:

\section{Definition 1.}

1. The sequence of functions $\left\{L_{m}\right\}$ is said to be uniformly bounded by $L$ if

$\exists L>0: \forall t>t_{1}, \forall m \in \mathbb{N}, \forall \tau_{m} \in \overline{\mathcal{T}_{m}(t)}, \quad\left|L_{m}\left(\tau_{m}, t\right)\right| \leq L$.

2. The sequence of functions $L_{m}$ is said to be exponentially bounded by $L$ if

$\exists L>0: \forall t>t_{1}, \forall m \in \mathbb{N}, \forall \tau_{m} \in \overline{\mathcal{T}_{m}(t)}, \quad\left|L_{m}\left(\tau_{m}, t\right)\right| \leq L^{m}$.

\section{Remark 1.}

1. If the sequence $\left\{L_{m}\right\}$ is uniformly bounded by $L$, it is also exponentially bounded by $\max \{L, 1\}$.

2. If the sequence is exponentially bounded by $L \leq 1$, it is also uniformly bounded by $L$.

\subsection{Computing the Value}

\subsubsection{Finite horizon}

We consider the problem with finite horizon $T$, and let $V_{1}$ be its value, i.e. the first forager's expected payoff. We aim to prove the following fact:

Theorem 1. If the sequence $\left\{L_{m}\right\}$ is exponentially bounded, then, the Value $V_{1}=\mathbb{E} J_{1}$ is given by

$V_{1}=\int_{t_{1}}^{T} \mathrm{e}^{-(\lambda+\delta)\left(t-t_{1}\right)} \sum_{m=1}^{\infty} \lambda^{m-1} M_{m}(t) \mathrm{d} t$.

Proof. We consider the same game, but where the maximum number $n$ of players that may arrive is known. In this game, let $J_{m}^{(n)}\left(\tau_{m}\right)$ be the payoff of the problem starting at the time $t_{m}$ of arrival of the $m$ th player, and $V_{m}^{(n)}\left(\tau_{m}\right)$ be its conditional expectation given $\tau_{m}$. We have for $m<n$ :

$J_{m}^{(n)}\left(\tau_{m}\right)= \begin{cases}\int_{t_{m}}^{t_{m+1}} \mathrm{e}^{-\delta\left(t-t_{m}\right)} L_{m}\left(\tau_{m}, t\right) \mathrm{d} t & \\ +\mathrm{e}^{-\delta\left(t_{m+1}-t_{m}\right)} J_{m+1}^{(n)}\left(\tau_{m}, t_{m+1}\right) & \text { if } \quad t_{m+1}<T, \\ \int_{t_{m}}^{T} \mathrm{e}^{-\delta\left(t-t_{m}\right)} L_{m}\left(\tau_{m}, t\right) \mathrm{d} t & \text { if } \quad t_{m}<T<t_{m+1}, \\ 0 & \text { if } \quad t_{m} \geq T .\end{cases}$

and

$V_{n}^{(n)}\left(\tau_{n}\right)=J_{n}^{(n)}\left(\tau_{n}\right)=\left\{\begin{array}{lll}\int_{t_{n}}^{T} \mathrm{e}^{-\delta\left(t-t_{n}\right)} L_{n}\left(\tau_{n}, t\right) \mathrm{d} t & \text { if } & t_{n}<T, \\ 0 & \text { if } & t_{n} \geq T .\end{array}\right.$

We now perform a calculation analogous to that in [11]. We want to evaluate the conditional expectation of $J_{m}^{(n)}$, given $\tau_{m}$. The random variables involved in this expectation are the $t_{k}$ for $k \geq m+1$. We isolate the variable $t_{m+1}$, with the exponential law of $t_{m+1}-t_{m}$. Because of the formula (3), we must distinguish the case where $t_{m+1} \leq T$ from the case where $t_{m+1}>T$, which happens with a probability $\exp \left(-\lambda\left(T-t_{m}\right)\right)$. As for the $t_{k}$ with higher indices $k$, we use the definition of the expectation given $\left(\tau_{m}, t_{m+1}\right)$ as $\mathbb{E} J_{m+1}^{(n)}\left(\tau_{m}, t_{m+1}\right)=V_{m+1}^{(n)}\left(t_{m}, t_{m+1}\right)$. We get

$$
\begin{aligned}
V_{m}^{(n)}\left(\tau_{m}\right)= & \int_{t_{m}}^{T} \lambda \mathrm{e}^{-\lambda\left(t_{m+1}-t_{m}\right)}\left[\int_{t_{m}}^{t_{m+1}} e^{-\delta\left(t-t_{m}\right)} L_{m}\left(\tau_{m}, t\right) \mathrm{d} t\right. \\
& \left.+\mathrm{e}^{-\delta\left(t_{m+1}-t_{m}\right)} V_{m+1}^{(n)}\left(\tau_{m}, t_{m+1}\right)\right] \mathrm{d} t_{m+1} \\
& +\mathrm{e}^{-\lambda\left(T-t_{m}\right)} \int_{t_{m}}^{T} \mathrm{e}^{-\delta\left(t-t_{m}\right)} L_{m}\left(\tau_{m}, t\right) \mathrm{d} t .
\end{aligned}
$$

Using Fubini's theorem, we get

$$
\begin{aligned}
V_{m}^{(n)}\left(\tau_{m}\right)= & \int_{t_{m}}^{T}\left[\int_{t}^{T} \lambda \mathrm{e}^{-\lambda\left(t_{m+1}-t_{m}\right)} \mathrm{d} t_{m+1}\right] \mathrm{e}^{-\delta\left(t-t_{m}\right)} L_{m}\left(\tau_{m}, t\right) \mathrm{d} t \\
& +\int_{t_{m}}^{T} \lambda \mathrm{e}^{-(\lambda+\delta)\left(t_{m+1}-t_{m}\right)} V_{m+1}^{(n)}\left(\tau_{m}, t_{m+1}\right) \mathrm{d} t_{m+1} \\
& +e^{-\lambda\left(T-t_{m}\right)} \int_{t_{m}}^{T} \mathrm{e}^{-\delta\left(t-t_{m}\right)} L_{m}\left(t_{m}, t\right) \mathrm{d} t .
\end{aligned}
$$

The inner integral in the first line above integrates explicitly, and its upper bound exactly cancels the last term in the last line. We also change the name of the integration variable of the second line from $t_{m+1}$ to $t$. We are left with

$V_{m}^{(n)}\left(\tau_{m}\right)=\int_{t_{m}}^{T} \mathrm{e}^{-(\lambda+\delta)\left(t-t_{m}\right)}\left[L_{m}\left(\tau_{m}, t\right)+\lambda V_{m+1}^{(n)}\left(\tau_{m}, t\right)\right] \mathrm{d} t$. 
We may now substitute formula (4) for $V_{2}^{(3)}$ in the same formula for $V_{1}^{(3)}$ :

$$
\begin{aligned}
V_{1}^{(3)}\left(t_{2}, t_{3}\right)= & \int_{t_{1}}^{T} \mathrm{e}^{-(\lambda+\delta)\left(t_{2}-t_{1}\right)}\left[L_{1}\left(t_{2}\right)+\lambda \int_{t_{2}}^{T} \mathrm{e}^{-(\lambda+\delta)\left(t_{3}-t_{2}\right)}\right. \\
& \left.\times\left(L_{2}\left(t_{2}, t_{3}\right)+\lambda \int_{t_{3}}^{T} L_{3}\left(t_{2}, t_{3}, t\right) \mathrm{d} t\right) \mathrm{d} t_{3}\right] \mathrm{d} t_{2} .
\end{aligned}
$$

Using again Fubini's theorem, we get

$$
\begin{aligned}
V_{1}^{(3)}\left(t_{2}, t_{3}\right)= & \int_{t_{1}}^{T} \mathrm{e}^{-(\lambda+\delta)\left(t-t_{1}\right)} L_{1}(t) \mathrm{d} t \\
& +\lambda \int_{t_{1}}^{T} \mathrm{e}^{-(\lambda+\delta)\left(t-t_{1}\right)}\left(\int_{t_{1}}^{t} L_{2}\left(t_{2}, t\right) \mathrm{d} t_{2}\right) \mathrm{d} t \\
& +\lambda^{2} \int_{t_{1}}^{T}\left[\int_{t_{1}}^{t}\left(\int_{t_{2}}^{t} \mathrm{e}^{-(\lambda+\delta)\left(t_{3}-t_{1}\right)} L_{3}\left(t_{1}, t_{2}, t_{3}, t\right) \mathrm{d} t_{3}\right) \mathrm{d} t_{2}\right] \mathrm{d} t
\end{aligned}
$$

We may now extend the same type of calculation to $V_{1}^{(n)}$. We find

$$
\begin{aligned}
V_{1}^{(n)}= & \sum_{m=1}^{n-1} \lambda^{m-1} \int_{t_{1}}^{T} \mathrm{e}^{-(\lambda+\delta)\left(t-t_{1}\right)} M_{m}(t) \mathrm{d} t+\lambda^{n-1} \\
& \times \int_{t_{1}}^{T} \int_{\mathcal{T}_{n}(t)} \mathrm{e}^{-(\lambda+\delta)\left(t_{n}-t_{1}\right)} L_{n}\left(\tau_{n}, t\right) \mathrm{d} \tau_{n} \mathrm{~d} t,
\end{aligned}
$$

or, equivalently, let

$\forall m<n, M_{m}^{(n)}=M_{m}, \quad M_{n}^{(n)}(t)=\int_{\mathcal{T}_{n}(t)} \mathrm{e}^{(\lambda+\delta)\left(t-t_{n}\right)} L_{n}\left(\tau_{n}, t\right) \mathrm{d} \tau_{n}$,

$V_{1}^{(n)}=\sum_{m=1}^{n} \lambda^{m-1} \int_{t_{1}}^{T} \mathrm{e}^{-(\lambda+\delta)\left(t-t_{1}\right)} M_{m}^{(n)}(t) \mathrm{d} \tau_{n}$.

Now, if the sequence $\left\{L_{m}\right\}$ is exponentially, respectively uniformly, bounded by $L$ (see Definition (1)), we get

$\left|M_{m}(t)\right| \leq \frac{\left(t-t_{1}\right)^{m-1}}{(m-1) !} L^{m}, \quad$ resp $\quad\left|M_{m}(t)\right| \leq \frac{\left(t-t_{1}\right)^{m-1}}{(m-1) !} L$,

and the last integral over $\mathcal{T}_{n}(t)$ in Eq. (5) is a fortiori less in absolute value than $\left|M_{n}(t)\right|$, since $L_{m}$ is multiplied by a factor less than 1.

We can now take the limit as $n \rightarrow \infty$. For each finite $n$, we have a sum $S_{n}$. Call $S_{n}^{\prime}$ the sum without the last term. The sum in (2) is $\lim _{n \rightarrow \infty} S_{n}^{\prime}$. Because of the remark above $S_{n}-S_{n}^{\prime} \rightarrow 0$ as $n \rightarrow \infty$. Hence $S_{n}$ and $S_{n}^{\prime}$ have the same limit as $n \rightarrow \infty$. Moreover, the estimation of $M_{m}(t)$ above implies that the series in Eq. (2) converges absolutely. Therefore the theorem is proved.

\section{Corollary 1.}

- If the sequence $\left\{L_{m}\right\}$ is exponentially bounded by $L$, then

$$
\begin{array}{lr}
\left|V_{1}\right| \leq \frac{L}{\lambda(L-1)-\delta}\left[\mathrm{e}^{[\lambda(L-1)-\delta]\left(T-t_{1}\right)}-1\right], & \text { if } \lambda(L-1)-\delta \neq 0, \\
\left|V_{1}\right| \leq\left(T-t_{1}\right) L & \text { if } \lambda(L-1)-\delta=0 .
\end{array}
$$

- If the sequence $\left\{L_{m}\right\}$ is uniformly bounded by $L$, then

$$
\begin{array}{ll}
\left|V_{1}\right| \leq \frac{L}{\delta}\left(\mathrm{e}^{-\delta\left(t-t_{1}\right)}-1\right) & \text { if } \delta \neq 0, \\
\left|V_{1}\right| \leq L\left(T-t_{1}\right), & \text { if } \delta=0 .
\end{array}
$$

- The above two inequalities become equalities if $L_{m}$ is constant equal to $L$.
It is worth mentioning that this also yields the value $V_{m}\left(\tau_{m}\right)$ for the $m$ th player arriving at time $t_{m}$ given the whole sequence of past arrival times $\tau_{m}$. We need extra notation:

$\forall n \geq m, \quad \tau_{m}^{n}=\left(t_{m+1}, \ldots, t_{n}\right), \quad \tau_{n}=\left(\tau_{m}, \tau_{m}^{n}\right)$,

$\mathcal{T}_{m}^{n}\left(t_{m}, t\right)=\left\{\tau_{m}^{n} \mid t_{m} \leq t_{m+1} \leq \ldots \leq t_{n} \leq t\right\}$,

and

$$
\begin{aligned}
M_{n}^{n}\left(\tau_{n}, t\right) & =L_{n}\left(\tau_{n}, t\right), \quad \forall n>m, M_{m}^{n}\left(\tau_{m}, t\right) \\
& =\int_{\tau_{m}^{n} \in \mathcal{T}_{m}^{n}\left(t_{m}, t\right)} L_{n}\left(\tau_{m}, \tau_{m}^{n}, t\right) \mathrm{d} \tau_{m}^{n} .
\end{aligned}
$$

Corollary 2. The value of the mth arriving player given the past sequence $\tau_{m}$ of arrival times is

$V_{m}\left(\tau_{m}\right)=\int_{t_{m}}^{T} \mathrm{e}^{-(\lambda+\delta)\left(t-t_{m}\right)} \sum_{k=m}^{\infty} \lambda^{k-m} M_{m}^{k}\left(\tau_{m}, t\right) \mathrm{d} t$.

\subsubsection{Infinite horizon}

We now tackle the problem of estimating the expectation of

$J_{1}=\int_{0}^{\infty} \mathrm{e}^{-\delta t} L_{m(t)}\left(\tau_{m}, t\right) \mathrm{d} t$.

(We have in mind a stationary problem, hence the choice of initial time 0 ). We will prove the following fact:

Theorem 2. If the sequence $\left\{L_{m}\right\}$ is uniformly bounded, or if it is exponentially bounded by $L$, and $\delta>\lambda(L-1)$, then the expectation $V_{1}$ of $J_{1}$ is given by

$V_{1}=\int_{0}^{\infty} \mathrm{e}^{-(\lambda+\delta) t} \sum_{m=1}^{\infty} \lambda^{m-1} M_{m}(t) \mathrm{d} t$.

Remark 2. If the sequence $\left\{L_{m}\right\}$ is exponentially bounded with $L$ $\leq 1$, the condition $\delta>\lambda(L-1)$ is automatically satisfied (and it is also uniformly bounded).

Proof. We start from the formula (2), set $t_{1}=0$, and denote the value with a superindex $(T)$ to note the finite horizon. This yields

$V_{1}^{(T)}=\int_{0}^{T} \mathrm{e}^{-(\delta+\lambda) t} \sum_{m=1}^{\infty} \lambda^{m-1} M_{m}(t) \mathrm{d} t$.

We only have now to check whether the integral converges as $T \rightarrow$ $\infty$. We use then the bounds (7), which show that

- If the sequence $\left\{L_{m}\right\}$ is exponentially bounded,

$$
\left|\sum_{m=1}^{\infty} \lambda^{m-1} M_{m}(t)\right| \leq L \mathrm{e}^{\lambda L t},
$$

- If the sequence $\left\{L_{m}\right\}$ is uniformly bounded,

$$
\left|\sum_{m=1}^{\infty} \lambda^{m-1} M_{m}(t)\right| \leq L \mathrm{e}^{\lambda t} \text {. }
$$

As a consequence, the integral in formula (9) converges as $T \rightarrow$ $\infty$, always if the $\left\{L_{m}\right\}$ are uniformly bounded, and if $\lambda(L-1)-\delta<$ 0 if they are exponentially bounded.

Corollary 3. If the sequence $\left\{L_{m}\right\}$ is exponentially bounded and $\delta>$ $\lambda(L-1)$, then

$\left|V_{1}\right| \leq \frac{L}{\delta-\lambda(L-1)}$,

if the sequence $\left\{L_{m}\right\}$ is uniformly bounded and $\delta>0$, then

$\left|V_{1}\right| \leq \frac{L}{\delta}$ 


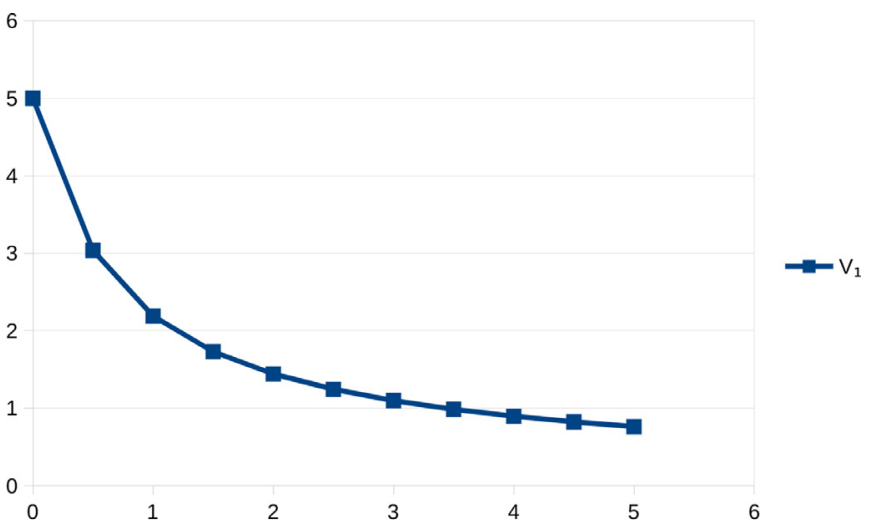

Fig. 1. The decrease of $V_{1}$ as $\lambda$ goes from 0 to 5 in the simple sharing problem, for $a=1, T-t_{1}=5$ and $\delta=0$.

if the $L_{m}$ are constant and $L_{m}\left(\tau_{m}, t\right)=L$, then

$V_{1}=\frac{L}{\delta}$

We also get the corresponding corollary:

Corollary 4. The expected payoff of the mth arriving player given the past sequence $\tau_{m}$ of arrival times is:

$V_{m}\left(\tau_{m}\right)=\int_{t_{m}}^{\infty} \mathrm{e}^{-(\lambda+\delta)\left(t-t_{m}\right)} \sum_{k=m}^{\infty} \lambda^{k-m} M_{m}^{k}\left(\tau_{m}, t\right) \mathrm{d} t$.

\section{Some examples}

\subsection{Simple sharing}

\subsubsection{The problem}

In this very simple application, we assume that a flux of the desirable good of $a$ units per time unit is available, say a renewable resource that regenerates at the constant rate of $a$ units per time unit, and the foragers present just share it equally. This example may fit biotrophic fungal plant parasites such as cereal rusts (see Appendix B).

\subsubsection{The Value}

Finite horizon. Thus, in this model (which is not accounted for by our theory [10]),

$\forall t, \forall m, \forall \tau_{m} \in \mathcal{T}_{m}(t), \quad L_{m}\left(\tau_{m}, t\right)=\frac{a}{m}$.

It follows that, in the finite horizon case,

$M_{m}(t)=a \frac{\left(t-t_{1}\right)^{m-1}}{m !}$.

Hence,

$$
\begin{aligned}
V_{1} & =a \int_{t_{1}}^{T} \mathrm{e}^{-(\lambda+\delta)\left(t-t_{1}\right)} \sum_{m=1}^{\infty} \frac{\lambda^{m-1}\left(t-t_{1}\right)^{m-1}}{m !} \\
& =a \int_{t_{1}}^{T} \frac{\mathrm{e}^{-(\lambda+\delta)\left(t-t_{1}\right)}}{\lambda\left(t-t_{1}\right)}\left(e^{\lambda\left(t-t_{1}\right)}-1\right) \mathrm{d} t .
\end{aligned}
$$

We immediately conclude:

Theorem 3. For the simple sharing problem in finite horizon, the value is

$V_{1}=a \int_{0}^{T-t_{1}} \mathrm{e}^{-\delta t} \frac{1-\mathrm{e}^{-\lambda t}}{\lambda t} \mathrm{~d} t$
We show in Fig. 1a graph of $V_{1}$ for $a=1, T=5$ and $\delta=0$ as a function of $\lambda$, for $\lambda \in[0,5]$. The integral was computed on a spreadsheet by the method of trapezes with a time step of .01 .

Infinite horizon. In infinite horizon, we may further use the identity

$\int_{0}^{\infty} \mathrm{e}^{-(\lambda+\delta) t} \frac{t^{m-1}}{(m-1) !} \mathrm{d} t=\frac{1}{(\lambda+\delta)^{m}}$

easily derived by successive integrations by parts. This immediately yields

$V_{1}=a \sum_{m=1}^{\infty} \frac{\lambda^{m-1}}{m(\lambda+\delta)^{m}}$.

We rearrange this expression as

$V_{1}=\frac{a}{\lambda} \sum_{m=1}^{\infty} \frac{1}{m}\left(\frac{1}{1+\frac{\delta}{\lambda}}\right)^{m}$.

Now, we use the identity, valid for $x \in(0,1)$,

$\sum_{m=1}^{\infty} \frac{x^{m}}{m}=-\ln (1-x)$

to obtain the following result:

Theorem 4. For the simple sharing problem in infinite horizon, the Value is

$V_{1}=\frac{a}{\lambda} \ln \left(1+\frac{\lambda}{\delta}\right)$

One can offer the following remarks:

\section{Remark 3.}

- As expected, when $\lambda \rightarrow 0, V_{1} \rightarrow a / \delta$, and $V_{1} \rightarrow 0$ when $\lambda \rightarrow \infty$.

- The derivative of $V_{1}$ with respect to $\lambda$ is always negative, increasing from $-a / 2 \delta^{2}$ for $\lambda=0$ to 0 as $\lambda \rightarrow \infty$.

- $V_{1}$ is decreasing with $\delta$, but diverges to infinity as $\delta \rightarrow 0$.

Finally, we get

Corollary 5. For the simple sharing problem in infinite horizon, the expected payoff of the mth forager arrived is

$$
\begin{aligned}
V_{m} & =\frac{a}{\lambda}\left(1+\frac{\delta}{\lambda}\right)^{m-1} \sum_{k=m}^{\infty} \frac{1}{k}\left(\frac{\lambda}{\lambda+\delta}\right)^{k} \\
& =\frac{a}{\lambda}\left(1+\frac{\delta}{\lambda}\right)^{m-1}\left[\ln \left(1+\frac{\lambda}{\delta}\right)-\sum_{k=1}^{m-1} \frac{1}{k}\left(1+\frac{\delta}{\lambda}\right)^{-k}\right] .
\end{aligned}
$$

A more general formula. At this stage, we have no explicit formula for the $m$ th arrived forager, $m>1$, in finite horizon. We give now two formulas, whose derivations can be found in A.1:

Theorem 5. For the simple sharing problem with horizon $T$, the expected reward of the mth arrived forager is given by any of the following formulas :

$$
\begin{aligned}
V_{m}^{(T)}\left(t_{m}\right)= & \frac{a}{\lambda+\delta} \mathrm{e}^{-(\lambda+\delta)\left(T-t_{m}\right)} \sum_{\ell=1}^{\infty} \frac{(\lambda+\delta)^{\ell}\left(T-t_{m}\right)^{\ell}}{\ell !} \\
& \times \sum_{k=0}^{\ell-1} \frac{1}{k+m}\left(1+\frac{\delta}{\lambda}\right)^{-k} .
\end{aligned}
$$


or

$$
\begin{aligned}
V_{m}^{(T)}\left(t_{m}\right)= & \frac{a}{\lambda}\left(1+\frac{\delta}{\lambda}\right)^{m-1}\left[\ln \left(1+\frac{\lambda}{\delta}\right)-\sum_{k=1}^{m-1} \frac{1}{k}\left(1+\frac{\delta}{\lambda}\right)^{-k}\right] \\
& -\frac{a}{\lambda+\delta} \mathrm{e}^{-(\lambda+\delta)\left(T-t_{m}\right)} \sum_{k=0}^{\infty} \frac{1}{k+m}\left(1+\frac{\delta}{\lambda}\right)^{-k} \\
& \times \sum_{\ell=0}^{k} \frac{(\lambda+\delta)^{\ell}\left(T-t_{m}\right)^{\ell}}{\ell !} .
\end{aligned}
$$

The first formula is easier to use for numerical computations, but the second one has the following properties:

\section{Remark 4.}

- The second term in the bracket of the first line cancels for $m=$ 1 , giving an alternate formula for $V_{1}$ to (11), (but probably less useful numerically).

- the second line goes to 0 as $T \rightarrow \infty$, allowing one to recover formulas (13) and, combining the two remarks (12).

\subsection{Harvesting a common: functional response of type 1}

\subsubsection{Notation}

Beyond the notation of the general theory, we have:

$x(t) \in \mathbb{R}_{+}$Available resource at time $t$.

$x_{1} \in \mathbb{R}_{+} \quad$ Initial amount of resource in the finite horizon problem.

$x_{0} \in \mathbb{R}_{+} \quad$ Initial amount of resource in the infinite horizon problem.

$a \in \mathbb{R}_{+} \quad$ Relative intake rate of all foragers: rate $=a x$.

$b \in \mathbb{R}_{+} \quad$ Relative renewal rate of the resource.

$c \in \mathbb{R}_{+} \quad c=b / a$ : if $m>c$, the resource goes down.

$\mu \in \mathbb{R}_{+} \quad \mu=\lambda / a$ a dimensionless measure of $\lambda$.

$\delta \in \mathbb{R}_{+} \quad$ Discount factor for the infinite horizon problem.

$v \in \mathbb{R}_{+} \quad v=\delta / a$. A dimensionless measure of $\delta$.

$\sigma_{m} \quad \sigma_{m}=\sum_{k=1}^{m} t_{k}$. A real random variable.

\subsubsection{The problem}

We consider a resource $x$ which has a "natural" growth rate $b$, (which may be taken equal to zero if desired), and decreases as it is harvested by the foragers. Each forager harvests a quantity ax of the resource per time unit. This is a functional response of type 1 in Holling's classification (see [15]), or "proportional harvesting", or "fixed effort harvesting" [23]. Assuming that this functional response does not change with predators density (no interference), we have

$\forall t \in\left(t_{m}, t_{m+1}\right), \quad \dot{x}=(b-m a) x, \quad x\left(t_{1}\right)=x_{1}$.

\subsubsection{Finite horizon}

We begin with a finite horizon $T$ and the payoff

$J_{1}=\int_{t_{1}}^{T} a x(t) \mathrm{d} t$.

Define

$\sigma_{m}=\sum_{k=1}^{m} t_{k}$.

With respect to the above theory, we have here

$$
\begin{aligned}
L_{m}\left(\tau_{m}, t\right) & =a \mathrm{e}^{(b-m a)\left(t-t_{m}\right)} \mathrm{e}^{[b-(m-1) a]\left(t_{m}-t_{m-1}\right)} \cdots \mathrm{e}^{(b-a)\left(t_{2}-t_{1}\right)} x_{1} \\
& =a \mathrm{e}^{b\left(t-t_{1}\right)} \mathrm{e}^{-a\left(m t-\sigma_{m}\right)} x_{1} .
\end{aligned}
$$

A more useful representation for our purpose is

$L_{m}\left(\tau_{m}, t\right)=\mathrm{e}^{(b-m a)\left(t-t_{m}\right)} L_{m-1}\left(\tau_{m-1}, t_{m}\right)$.
It follows that

$$
M_{m}(t)=\int_{t_{1}}^{t} \mathrm{e}^{(b-m a)\left(t-t_{m}\right)} M_{m-1}\left(t_{m}\right) \mathrm{d} t_{m}, \quad M_{1}(t)=a \mathrm{e}^{(b-a)\left(t-t_{1}\right)} x_{1} .
$$

Lemma 1. The solution of the recursion (17) is

$M_{m}(t)=\frac{a \mathrm{e}^{(b-a)\left(t-t_{1}\right)}}{(m-1) !}\left(\frac{1-\mathrm{e}^{-a\left(t-t_{1}\right)}}{a}\right)^{m-1} x_{1}$.

Proof. Notice first that the formula is correct for $M_{1}(t)=$ $a \exp \left[(b-a)\left(t-t_{1}\right)\right] x_{1}$. From Eq. (17), we derive an alternate recursion:

$\frac{\mathrm{d}}{\mathrm{d} t} M_{m+1}(t)=[b-(m+1) a] M_{m+1}(t)+M_{m}(t), \quad M_{m+1}\left(t_{1}\right)=0$.

It is a simple matter to check that the formula derived from (18) for $M_{m+1}$ :

$M_{m+1}(t)=\frac{a \mathrm{e}^{(b-a)\left(t-t_{1}\right)}}{m !}\left(\frac{1-\mathrm{e}^{-a\left(t-t_{1}\right)}}{a}\right)^{m} x_{1}$

together with (18) does satisfy that recursion.

From the above lemma, we derive the following:

Corollary 6. For the proportional foraging game, we have

$\sum_{m=1}^{\infty} \lambda^{m-1} M_{m}(t)=a \exp \left[(b-a)\left(t-t_{1}\right)+\frac{\lambda}{a}\left(1-\mathrm{e}^{-a\left(t-t_{1}\right)}\right)\right] x_{1}$.

Substituting this result in (2), we obtain:

Theorem 6. The value of the finite horizon proportional harvesting problem is as follows: let

$\frac{b}{a}=c, \quad \frac{\lambda}{a}=\mu$

then

$V_{1}=a \mathrm{e}^{\mu} \int_{t_{1}}^{T} \exp \left[(c-\mu-1) a\left(t-t_{1}\right)-\mu \mathrm{e}^{-a\left(t-t_{1}\right)}\right] \mathrm{d} t x_{1}$.

or equivalently

$V_{1}=\mathrm{e}^{\mu} \sum_{k=0}^{\infty} \frac{(-\mu)^{k}}{k !} \frac{1}{\mu-c+k+1}\left(1-\mathrm{e}^{-(\mu-c+k+1) a\left(T-t_{1}\right)}\right) x_{1}$.

Proof. It remains only to derive the alternate, second, formula. It is obtained by expanding $\exp \left[\left(-\mu \exp \left(-a\left(t-t_{1}\right)\right)\right]\right.$ into its power series expansion to obtain

$V_{1}=a \mathrm{e}^{\mu} \sum_{k=0}^{\infty} \frac{(-\mu)^{k}}{k !} \int_{t_{1}}^{T} \mathrm{e}^{(c-\mu-k-1) a\left(t-t_{1}\right)} \mathrm{d} t x_{1}$

and integrate each term.

Remark 5. Remark that, although it looks awkward, formula (19) is numerically efficient, as, an alternating exponential-like series, it converges very quickly.

\subsubsection{Infinite horizon}

It is easy to derive from there the infinite horizon case with the same dynamics, $x(0)=x_{0}$, and

$J_{1}=a \int_{0}^{\infty} \mathrm{e}^{-\delta t} x(t) \mathrm{d} t$

Theorem 7. The Value of the infinite horizon proportional harvesting problem is finite if and only if $\delta>b-a-\lambda$. In that case it is as follows: let

$\frac{b}{a}=c, \quad \frac{\lambda}{a}=\mu, \quad \frac{\delta}{a}=v$, 


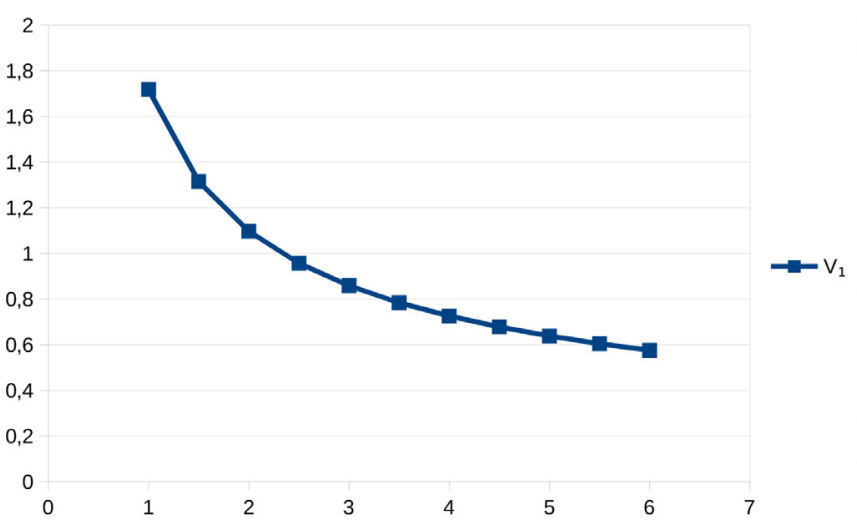

Fig. 2. The decrease of $V_{1}$ as $\lambda$ increases, here as a function of $\mu=\lambda / a$, for the infinite horizon proportional foraging problem. $c=2, v=1, x_{0}=1$.

then

$V_{1}=a \mathrm{e}^{\mu} \int_{0}^{\infty} \exp \left[(c-\mu-v-1) a t-\mu \mathrm{e}^{-a t}\right] \mathrm{d} t x_{0}$,

or equivalently

$V_{1}=\mathrm{e}^{\mu} \sum_{k=0}^{\infty} \frac{(-\mu)^{k}}{k !} \frac{1}{\mu+v-c+k+1} x_{0}$

or equivalently

$V_{1}=\mathrm{e}^{\mu} \int_{0}^{1} z^{\mu+\nu-c} \mathrm{e}^{-\mu z} \mathrm{~d} z x_{0}$.

Proof. The first formula above is obtained by carrying the integration from 0 to $\infty$ instead of from $t_{1}$ to $T$. The second formula follows as in the finite horizon case, and the third one, numerically more useful, is obtained by considering the function

$F(z)=\mathrm{e}^{\mu} \sum_{k=0}^{\infty} \frac{(-\mu)^{k}}{k !} \frac{z^{\mu+\nu-c+k+1}}{\mu+v-c+k+1} x_{0}$,

Clearly, $F(1)=V_{1}$. Moreover, the condition of the theorem that $\delta>b-a-\lambda$ translates into $\mu+v-c+1>0$, so that all powers of $z$ in $F(z)$ are positive, and hence $F(0)=0$. Now, differentiate the terms of the series, to obtain

$\mathrm{e}^{\mu} z^{\mu+\nu-c} \sum_{k=0}^{\infty} \frac{(-\mu z)^{k}}{k !}=\mathrm{e}^{\mu} z^{\mu+\nu-c} \mathrm{e}^{-\mu z}$.

This is always a convergent series. Hence it represents the derivative $F^{\prime}(z)$ of $F(z)$. As a consequence, we have

$V_{1}=\int_{0}^{1} F^{\prime}(z) \mathrm{d} z=\mathrm{e}^{\mu} \int_{0}^{1} z^{\mu+\nu-c} \mathrm{e}^{-\mu z} \mathrm{~d} z x_{0}$.

If $\mu+v-c$ happens to be a positive integer, a very unlikely fact in any real application, successive integrations by parts yield a closed form expression for the integral formula (21).

Remark 6. Clearly, the same remark as in the finite horizon case holds for formula (20). Formula (21) is even numerically easier to implement. A slight difficulty appears if $\mu+v-c<0$. Remember, though, that it must anyhow be larger than -1 . In that case, $F^{\prime}(0)=\infty$. One may integrate from a small value $z_{0}$, using the first term of the series (22), $F\left(z_{0}\right) \simeq e^{\mu} z_{0}^{\mu+\nu-c+1}$, as a good approximation of $F$.

We show in Fig. 2a graph of $V_{1}$ as a function of $\mu$, for $\mu \in[1$, $6]$, for the infinite horizon problem, obtained with formula (21), for $c=2, v=1, x_{0}=1$. The integral was computed on a spreadsheet, with the formula of trapezes, with a step size of .01 .
3.3. Harvesting a common: functional response of type 2

\subsubsection{Notation}

Beyond the notation of the general theory, we have:

$x \in \mathbb{R}_{+} \quad$ Available resource.

$x_{1} \in \mathbb{R}_{+} \quad$ Initial amount of resource.

$a \in \mathbb{R}_{+} \quad$ Coefficient of the intake rate.

$\alpha \in(0,1)$ Power parameter of the intake rate $=a x^{1-\alpha}$.

$p=\frac{1}{\alpha}-1 \quad$ Other parametrization of $\alpha=1 /(1+p)$.

$h \in \mathbb{R}^{+} \quad$ Duration until exhaustion of the resource if the first forager remains alone.

$q \in \mathbb{R}_{-} \quad$ Logarithm of the probability that the first forager remain alone until exhaustion of the resource.

\subsubsection{A family of concave functional responses}

In this example, we assume a non renewable resource, and foragers or predators with a concave functional response. Specifically, if the resource amount is $x$, the intake rate of a forager is assumed to be $a x^{(1-\alpha)}, \alpha \in(0,1)$. This provides us with a one-parameter family ${ }^{1}$ of concave functional responses resembling Holling's type 2. By contrast to the laws most commonly used, such as the Michaelis-Menten harmonic law ax/(1+hax) [23], and to the curves shown by Holling [15], they lack a plateau at large densities. A distinctive feature is their vertical tangent (infinite derivative) at the origin. ${ }^{2}$ It has the nice consequence that it makes the resource go to zero in finite time, certainly a more realistic feature than an infinite tail with very low resource left, mainly so if the resource is discrete (number of hosts parasitized, of preys eaten, ....). ${ }^{3}$ This makes the problem naturally with a finite horizon, and limits to a very small number the probability of having a very large number of foragers participating, although it is not bounded a priori. ${ }^{4}$

In short, our model is

- less realistic than the harmonic law at high prey densities,

- more realistic than the harmonic law at small and vanishing prey densities.

We therefore have:

$\forall t \in\left[t_{m}, t_{m+1}\right], \quad \dot{x}=-\max ^{1-\alpha}, \quad x\left(t_{1}\right)=x_{1}$.

The dynamics (23) immediately integrate into: $\forall t \in\left[t_{m}, t_{m+1}\right]$,

$x^{\alpha}(t)=\left\{\begin{array}{lll}x^{\alpha}\left(t_{m}\right)-\alpha \operatorname{am}\left(t-t_{m}\right) & \text { if } & t \leq \frac{x^{\alpha}\left(t_{m}\right)}{\alpha a m}+t_{m}, \\ 0 & \text { if } \quad t \geq \frac{x^{\alpha}\left(t_{m}\right)}{\alpha a m}+t_{m} .\end{array}\right.$

Applying this to the first stage $m=1$, we are led to introduce the two parameters

$h=\frac{x_{1}^{\alpha}}{\alpha a} \quad$ and $\quad q=-\lambda h$

which are respectively the maximum possible duration of the harvesting activity, assuming a lone forager, and the logarithm of the probability that the first forager be actually left alone during that time. We also introduce two more useful quantities:

$p=\frac{1}{\alpha}-1$,

\footnotetext{
1 The parameter $a$ amounts to a simple rescaling of time. The notation $x^{1-\alpha}$ was preferred to $x^{\alpha}$ because it simplifies later calculations.

${ }^{2}$ Holling [15] does not give explicit mathematical formulas for the various functional responses. But we notice that in his Fig. 8, he seems to show a vertical tangent at the origin for type 2 .

3 This avoids the paradox of the "atto fox" [20].

4 A somewhat unpleasant consequence of this law is that, if the dimension of the resource $x$ is $X$, the dimension of $a$ is $X^{\alpha} T^{-1}$.
} 
so that $\alpha=1 /(1+p)$, and

$\forall r \in \mathbb{R}_{+}, \forall \ell \in \mathbb{N}, \ell \geq 2, \quad P_{r}(1)=1, \quad P_{r}(\ell)=\prod_{i=1}^{\ell-1}(r+i)$.

\subsubsection{Expected reward}

With these notation we can state the following fact, whose proof is given in A.2.

Theorem 8. For the problem of harvesting a nonrenewable resource with a functional response $L(x)=a x^{p /(p+1)}, p$ a positive real number, using $q$ the natural logarithm of the probability that the first forager remains alone until exhaustion of the resource, and notation (26),

$V_{m+1}=x_{m+1} \sum_{\ell=1}^{\infty} \frac{q^{\ell-1}}{P_{p+1}(\ell)} \sum_{n=1}^{\ell} \frac{(-1)^{\ell-n}}{(\ell-n) !} \sum_{k=1}^{n} \frac{1}{k !(m+k)^{n-k}}$.

Remark 7. Several remarks are in order.

- The expected reward of the first forager is therefore obtained by placing $m=0$ in the above formula.

- The first term of the power series in $q$ is just 1 . Therefore this could be written

$V_{1}=x_{1}[1+q S]$

where $S$ is a power series in $q$. We therefore find that if no other forager may come, i.e. $\lambda=0$, hence $q=0$, we recover $V_{1}=x_{1}$ : the lone forager gets all the resource. And since $q<$ $0, V_{1}$ is less than $x_{1}$ as soon as $q \neq 0$.

- The series converges, and even absolutely. As a matter of fact, it is easy to see that the last two, finite, sums are less than $\left(\mathrm{e}^{2}+\right.$ $1) / 2$. (Taking the sum of the positive terms only and extending them to infinity.)

- The case $p=1$ (i.e. $\alpha=1 / 2$ ) is somewhat simpler. In particular, in that case, $P_{p}(\ell)=\ell !$, and $P_{p+1}(\ell)=(\ell+1) ! / 2$.

\subsubsection{Numerical computation}

We aim to show that, in spite of the unappealing aspect of our formulas, they are indeed very easy to implement numerically. We focus first on the payoff of the first player, i.e. formula (27) with $m=0$.

On the one hand, $q$ being negative, it is an alternating series, converging quickly: the absolute value of the remainder is less than that of the first term neglected, and a fortiori than that of the last term computed. Therefore it is easy to appreciate the precision of a numerical computation. The smaller the intensity $\lambda$ of the Poisson process of arrivals, the faster the series converges. On the other hand, it lends itself to an easy numerical implementation along the following scheme. Let

$V_{1}=x_{1} \sum_{\ell=1}^{\infty} u_{\ell} \sum_{n=1}^{\ell} v_{\ell-n} w_{n}, \quad w_{n}=\sum_{k=1}^{n} y_{n, k}$

Obviously,

$u_{\ell}=\frac{q^{\ell-1}}{P_{p+1}(\ell)}, \quad v_{n}=\frac{(-1)^{n}}{n !}, \quad y_{n, k}=\frac{1}{k ! k^{n-k}}$.

Therefore, the following recursive scheme is possible :

$\begin{array}{ll}u_{1}=1, \quad \forall \ell>1, & u_{\ell}=\frac{q u_{\ell-1}}{p+\ell}, \\ v_{0}=1, \quad \forall n>1, & v_{n}=\frac{-v_{n-1}}{n},\end{array}$

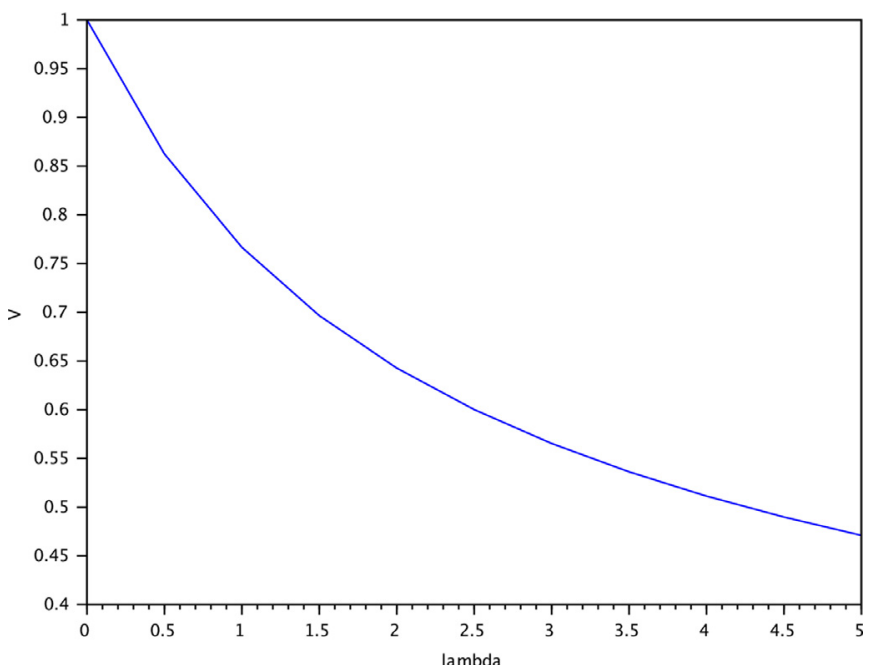

Fig. 3. The decrease of $V_{1}$ as $\lambda$ increases from 0 to 5 for the functional response of type 2 , with $x_{1}=1, a=1, \alpha=.5$.

$$
\begin{aligned}
y_{1,1}=1, \quad \forall n>1,1 \leq k<n, \quad y_{n, k} & =\frac{y_{n-1, k}}{k}, \\
\forall n>1, \quad y_{n, n} & =\frac{y_{n-1, n-1}}{n} .
\end{aligned}
$$

The sequences $\left\{u_{n}\right\},\left\{v_{n}\right\}$ and $\left\{w_{n}\right\}$ need be computed only once. Altogether, if we want to compute $L$ steps, there are $2 L^{2}+3(L-1)$ arithmetic operations to perform. A small task on a modern computer even for $L=\mathrm{a}$ few hundreds. Moreover, given the factorials, it seems unlikely that more than a few tens of coefficients be ever necessary, as our limited numerical experiments show. In that respect, the computational scheme we have proposed is adapted to the case where $|q|$ is large ( $\lambda$ large), lumping the numerator $q^{\ell-1}$ with the denominator $P_{p+1}(\ell)$-which is larger than $\ell !-$ to avoid multiplying a very large number by a very small one, a numerically ill behaved operation. For small $|q|$ (say, no more than one), one may compute a few coefficients of the power series separately, and then use the polynomial to compute $V_{1}$ for various values of $q$.

We show in Fig. 3a plot of $V_{1}$ as a function of $\lambda$, for $\alpha=.5, a=$ 1 and $x_{1}=1$. (i.e. a time to exhaustion of the resource for a lone forager equal to two.) It was obtained using the recursive scheme above (in Scilab). For the larger $\lambda=5$, we needed 40 terms in the infinite sum (which was also sufficient for $\lambda=6$ ).

Finally, formula (27), giving the expected payoff of an agent arriving when $m$ other agents are present, can clearly be implemented numerically along the same scheme as previously, replacing $y_{n, k}=y_{n-1, k} / k$ by $y_{n, k}=y_{n-1, k} /(m+k)$.

\section{Conclusion}

The problem considered here is only that of evaluating the expected reward of these identical agents arriving as a Poisson random process. This seems to be a prerequisite to many problems in foraging theory in that reasonably realistic framework, although it was avoided in the investigation of the optimal patch leaving strategies [10]. (Notice, however, that our first example is not accounted for by that theory.) In that respect, we were able to build a rather general theory, independent of the particular functional response of the foragers and of the resource depletion/renewal mechanism. A nice feature of the theory is that, while the complete data of the problem involve the sequence of functions $L_{m}\left(\tau_{m}\right.$, $t$ ), each a function of $m$ real variables, a very large amount of data, the result only depends on the sequence of functions of one real variable $M_{m}(t)$. 
Three particular examples, of increasing complexity, all three in scramble competition (no interference), show that this general theory can be particularized to more specific contexts, leading to efficient numerical algorithms, if not always to closed formulas. We provide numerical results that do not claim to be representative of any real situation, but are meant to show that the computation can easily be performed, and to display the qualitative aspect of the results. The first two examples: simple, equal, sharing of a constant flux of goods and proportional foraging of a (slowly) renewable resource, can easily be implemented on a spreadsheet. The example with a functional response of Holling type 2 requires a small program, that runs essentially instantly. In all three cases, the decrease of $V_{1}$ as $\lambda$ increases look qualitatively similar.

However, we need, as in our examples, to be able to integrate explicitly in closed form the dynamic equation of the resource to compute the $M_{m}$ sufficiently explicitly. This is why we chose the particular functional response we proposed as an approximation of a Holling type 2 response. Admittedly a major weakness of this theory so far. What would be needed would be a theory exploiting the simple, sequential nature of the Markov process at hand, but not dependent on an explicit integration of the differential dynamics. An open problem at this stage.

Also, we are far from determining any kind of optimal behavior, such as diet choice as in [11]. But this study was a first necessary step in characterizing the efficiency of the foraging process. Since we have the result for each number of agents present upon joining, it may be useful to decide whether an individual should join [25]. Other exciting possibilities for future research include exploring further the Simple sharing example, which may provide original insights as to the evolution of the latent period in plant parasites (Appendix B).

\section{Appendix A. Proofs}

\section{A.1. Proof of Theorem 5}

We aim to derive formulas (14) and (15). We shall simplify the notation through the use of

$\alpha:=\lambda+\delta, \quad$ and $\quad \theta:=T-t_{m}$.

We start from

$$
\begin{aligned}
V_{m} & =a \int_{t_{m}}^{T} \mathrm{e}^{-\alpha \theta} \sum_{k=m}^{\infty} \frac{\lambda^{k-m}\left(t-t_{m}\right)^{k-m}}{k(k-m) !} \mathrm{d} t \\
& =\frac{a}{\lambda^{m}} \sum_{k=m}^{\infty} \frac{\lambda^{k}}{k} \int_{0}^{\theta} \mathrm{e}^{-\alpha t} \frac{t^{k-m}}{(k-m) !} \mathrm{d} t .
\end{aligned}
$$

Successive integrations by parts show that

$\forall n \in \mathbb{N}, \quad \int_{0}^{\theta} \mathrm{e}^{-\alpha t} \frac{t^{n}}{n !}=\frac{1}{\alpha^{n+1}}\left(1-\mathrm{e}^{-\alpha \theta} \sum_{\ell=0}^{n} \frac{\alpha^{\ell} \theta^{\ell}}{\ell !}\right)$.

Substitute this in the previous formula, to get

$$
\begin{aligned}
V_{m} & =a \frac{\alpha^{m-1}}{\lambda^{m}}\left[\sum_{k=m}^{\infty} \frac{\lambda^{k}}{k \alpha^{k}}-\mathrm{e}^{-\alpha \theta} \sum_{k=m}^{\infty} \frac{\lambda^{k}}{k \alpha^{k}} \sum_{\ell=0}^{k-m} \frac{\alpha^{\ell} \theta^{\ell}}{\ell !}\right] \\
& =a \frac{\alpha^{m-1}}{\lambda^{m}}\left[\sum_{k=m}^{\infty} \frac{\lambda^{k}}{k \alpha^{k}}-\mathrm{e}^{-\alpha \theta} \sum_{\ell=0}^{\infty} \frac{\alpha^{\ell} \theta^{\ell}}{\ell !} \sum_{k=\ell+m}^{\infty} \frac{\lambda^{k}}{k \alpha^{k}}\right] .
\end{aligned}
$$

We use twice the identity

$$
\forall n \in \mathbb{N}, \quad \sum_{k=n}^{\infty} \frac{\lambda^{k}}{k \alpha^{k}}=\ln \left(1+\frac{\lambda}{\delta}\right)-\sum_{k=1}^{n-1} \frac{\lambda^{k}}{k \alpha^{k}} .
$$

The two terms $\ln (1+\lambda / \delta)$ cancel each other, and we are left with

$$
V_{m}=a \frac{\alpha^{m-1}}{\lambda^{m}}\left[-\sum_{k=1}^{m-1} \frac{\lambda^{k}}{k \alpha^{k}}+\mathrm{e}^{-\alpha \theta} \sum_{\ell=0}^{\infty} \frac{\alpha^{\ell} \theta^{\ell}}{\ell !} \sum_{k=1}^{\ell+m-1} \frac{\lambda^{k}}{k \alpha^{k}}\right] \text {. }
$$

We write the last sum over $k$ as a sum from $k=1$ to $m-1$ plus a sum from $k=m$ to $\ell+m-1$. The first one cancels with the first sum in the square bracket, to yield

$V_{m}=a \frac{\alpha^{m-1}}{\lambda^{m}} e^{-\alpha \theta} \sum_{\ell=1}^{\infty} \frac{\alpha^{\ell} \theta^{\ell}}{\ell !} \sum_{k=m}^{\ell+m-1} \frac{\lambda^{k}}{h \alpha^{k}}$.

It suffices to re-introduce the leading factor $(\alpha / \lambda)^{m-1}$ in the last sum and to shift the summation index $k$ by $m$ to obtain formula (14).

To obtain formula (15), interchange the order of summation in the above double sum (all the series involved are absolutely convergent):

$V_{m}=a \frac{\alpha^{m-1}}{\lambda^{m}} e^{-\alpha \theta} \sum_{k=m}^{\infty} \frac{\lambda^{k}}{k \alpha^{k}} \sum_{\ell=k-m+1}^{\infty} \frac{\alpha^{\ell} \theta^{\ell}}{\ell !}$,

and substitute

$\sum_{\ell=k-m+1}^{\infty} \frac{\alpha^{\ell} \theta^{\ell}}{\ell !}=\mathrm{e}^{\alpha \theta}-\sum_{\ell=0}^{k-m} \frac{\alpha^{\ell} \theta^{\ell}}{\ell !}$

to get

$V_{m}=a \frac{\alpha^{m-1}}{\lambda^{m}}\left[\ln \left(1+\frac{\alpha}{\delta}\right)-\sum_{k=1}^{m-1} \frac{\lambda^{k}}{k \alpha^{k}}-\mathrm{e}^{-\alpha \theta} \sum_{k=m}^{\infty} \frac{\lambda^{k}}{k \alpha^{k}} \sum_{\ell=0}^{k-m} \frac{\alpha^{\ell} \theta^{\ell}}{\ell !}\right]$.

Transform the last term in the square bracket as we did to get formula (14) to obtain formula (15).

\section{A.2. Proof of Theorem 8}

Expected reward of the first player. We aim to derive formula (27). For simplicity, we start with the expected reward of the first player, i.e. $m=0$ in that formula. We remind the reader of notation (25), and we will use the extra notation

$b=a^{1+p} \alpha^{p}, \quad$ and $\quad \sigma_{m}=\sum_{k=1}^{m} t_{k}$.

We remark that

$b h^{1+p}=\frac{x_{1}}{\alpha}=(1+p) x_{1}$.

Now, in formula (24), replace $x^{\alpha}\left(t_{m}\right)$ by its formula, taken again in (24) but for $t \in\left[t_{m-1}, t_{m}\right]$. To take care of the possibility that zero be reached, we introduce a notation for the positive part:

$\llbracket[X]_{+}=\max \{X, 0\}$.

We obtain

$x^{\alpha}(t)=\llbracket\left[x^{\alpha}\left(t_{m-1}\right)-\alpha a\left[(m-1)\left(t_{m}-t_{m-1}\right)+m\left(t-t_{m}\right)\right] \rrbracket_{+}\right.$.

Iterate this process until expressing $x^{\alpha}(t)$ in terms of $x^{\alpha}\left(t_{1}\right)$ and finally use notation (16) to obtain

$$
\begin{aligned}
\chi^{\alpha}(t)= & \llbracket \alpha a\left[h-\left(t_{2}-t_{1}\right)-2\left(t_{3}-t_{2}\right)-\cdots\right. \\
& \left.-(m-1)\left(t_{m}-t_{m-1}\right)-m\left(t-t_{m}\right)\right] \rrbracket_{+} \\
= & \alpha a\left[h+\sigma_{m}-m t \rrbracket_{+} .\right.
\end{aligned}
$$

As a consequence,

$L_{m}\left(\tau_{m}, t\right)=b \llbracket h+\sigma_{m}-m t \rrbracket_{+}^{p}$.

To keep the formulas readable, we use the notation (26).

We are now in a position to state the following fact: 
Proposition 2. For $L_{m}$ given by (A.2) with (16), $M_{m}$ as defined in (1) is given by $M_{1}(t)=b[h-t]_{+}^{p}$, and for $m \geq 2$ :

$M_{m}(t)=\frac{b}{P_{p}(m)} \sum_{k=1}^{m} \frac{(-1)^{k-1}}{(m-k) !(k-1) !} \llbracket h-k\left(t-t_{1}\right) \rrbracket_{+}^{p+m-1}$.

Proof. Observe that the formula is correct for $m=1$. Then, we need the simple lemma:

Lemma 2. Let a real number $b$, two real numbers $u<v$, and two positive real numbers $\ell$ and $r$ be given. Then

$\int_{u}^{v} \llbracket b+\ell s \rrbracket_{+}^{r} \mathrm{~d} s=\frac{1}{\ell(r+1)}\left(\llbracket b+\ell v \rrbracket_{+}^{r+1}-\llbracket\left[b+\ell u \rrbracket_{+}^{r+1}\right)\right.$.

Proof of the lemma. If $b+\ell v \leq 0$, then clearly the integrand is always zero, thus so is the integral, as well as our right hand side (r.h.s.). If $b+\ell u \geq 0$, the integrand is always positive, as well as the two terms of the r.h.s. This is a simple integration. If $u<-\ell / b<v$, we should integrate from $-\ell / b$ to $v$. Then the lower bound corresponds to $b+\ell s=0$, and we only have the term corresponding to the upper bound, which is what our r.h.s. gives.

We use the following representation of $M_{m}$ :

$M_{m}(t)=\int_{t_{1}}^{t} \mathrm{~d} t_{m} \int_{t_{1}}^{t_{m}} \mathrm{~d} t_{m-1} \int_{t_{1}}^{t_{m-1}} \mathrm{~d} t_{m-2} \ldots \int_{t_{1}}^{t_{3}} \llbracket h+\sigma_{m}-m t \rrbracket_{+}^{p} \mathrm{~d} t_{2}$.

To carry out this calculation, we introduce, for $m \geq 2$ the kernel

$$
\begin{aligned}
K_{m}\left(t_{m}, t\right)= & \frac{1}{P_{p}(m-1)} \sum_{k=1}^{m-1} \frac{(-1)^{k-1}}{(m-k-1) !(k-1) !} \\
& \times\left[h+k t_{1}+(m-k) t_{m}-m t\right]^{p+m-2},
\end{aligned}
$$

and the quantity

$N_{m}\left(t_{m+1}, t\right)=\int_{t_{1}}^{t_{m+1}} K_{m}\left(t_{m}, t\right) \mathrm{d} t_{m}$.

We first notice that $M_{2}$ is obtained by taking the positive parts of all terms in $b N_{2}(t, t)$. To get $M_{3}(t)$ we may simply replace everywhere $h$ by $h+t_{3}-t$, which behaves as a constant in previous integrations, integrate in $t_{3}$ from $t_{1}$ to $t$, and take the positive parts of all terms. And so on. It remains to derive the general forms of $K_{m}$ and $N_{m}$ by induction. Let us start from the formula given for $K_{m}$. Integrating, we get

$$
\begin{aligned}
& N_{m}\left(t_{m+1}, t\right) \\
& =P_{p}(m)^{-1} \sum_{k=1}^{m-1} \frac{(-1)^{k-1}}{(m-k) !(k-1) !} \\
& \quad \times\left[\left(h+k t_{1}+(m-k) t_{m+1}-m t\right)^{p+m-1}-\left(h+m t_{1}-m t\right)^{p+m-1}\right] .
\end{aligned}
$$

The second term in the r.h.s. is independent from $k$. Recognizing combinatorial coefficients, and using $(1+(-1))^{m-1}=0$, we get

$$
\begin{aligned}
\sum_{k=1}^{m-1} \frac{(-1)^{k}}{(m-k) !(k-1) !} & =\frac{-1}{(m-1) !} \sum_{k=0}^{m-2}(-1)^{k}\left(\begin{array}{c}
m-1 \\
k
\end{array}\right) \\
& =\frac{(-1)^{m-1}}{(m-1) !},
\end{aligned}
$$

which we can include as the term $k=m$ of the sum, leading to

$$
\begin{aligned}
N_{m}\left(t_{m+1}, t\right)= & \frac{1}{P_{p}(m)} \sum_{k=1}^{m} \frac{(-1)^{k-1}}{(m-k) !(k-1) !} \\
& \times\left[h+k t_{1}-(m-k) t_{m+1}-m t\right]^{m} .
\end{aligned}
$$

Taking the positive part of each term in $\left(a^{2} / 2\right) N_{m}(t, t)$ yields formula (A.3), and adding $t_{m+1}-t$ to all terms yields formula analogous to (A.4) for $K_{m+1}$, proving the proposition.
We therefore have

$$
\begin{aligned}
V_{1}= & b \sum_{m=1}^{\infty} \frac{\lambda^{m-1}}{P_{p}(m)} \sum_{k=1}^{m} \frac{(-1)^{k-1}}{(m-k) !(k-1) !} \\
& \times \int_{t_{1}}^{h+t_{1}}\left[h-k\left(t-t_{1}\right) \rrbracket_{+}^{p+m-1} \mathrm{e}^{-\lambda\left(t-t_{1}\right)} \mathrm{d} t\right. \\
= & b \sum_{m=1}^{\infty} \frac{\lambda^{m-1}}{P_{p}(m)} \sum_{k=1}^{m} \frac{(-1)^{k-1}}{(m-k) !(k-1) !} \int_{0}^{h / k}(h-k t)^{p+m-1} \mathrm{e}^{-\lambda t} \mathrm{~d} t .
\end{aligned}
$$

Using the power expansion of $\exp (-\lambda t)$, and then successive integrations by parts, we find

$$
\begin{aligned}
\int_{0}^{h / k}(h-k t)^{p+m-1} \mathrm{e}^{-\lambda t} \mathrm{~d} t & =\sum_{\ell=0}^{\infty} \int_{0}^{h / k}(h-k t)^{p+m-1} \frac{(-\lambda t)^{\ell}}{\ell !} \mathrm{d} t \\
& =\theta^{p+m} \sum_{\ell=0}^{\infty} \frac{(-\lambda h)^{\ell}}{k^{\ell+1}} \prod_{i=m}^{m+\ell}(p+i)^{-1}
\end{aligned}
$$

We substitute this expression in the formula for $M_{m}(t)$, regroup powers of $\lambda h$ and products $p+i$ :

$V_{1}=\frac{b h^{p}}{\lambda} \sum_{m=1}^{\infty} \sum_{k=1}^{m} \frac{(-1)^{k+m-1}}{(m-k) !(k-1) !} \sum_{\ell=m}^{\infty} \frac{(-\lambda h)^{\ell}}{k^{\ell-m+1} P_{p}(\ell+1)}$.

We take a factor $\lambda h$ out, change the order of summation and use (A.1) to find

$V_{1}=x_{1} \sum_{\ell=1}^{\infty} \frac{q^{\ell-1}}{P_{p+1}(\ell)} \sum_{m=1}^{\ell} \sum_{k=1}^{m} \frac{(-1)^{m+k}}{(m-k) ! k ! k^{\ell-m}}$.

Finally, let $n=\ell-m+k$ replace $m$ as a summation index. We obtain:

$V_{1}=x_{1} \sum_{\ell=1}^{\infty} \frac{q^{\ell-1}}{P_{p+1}(\ell)} \sum_{n=1}^{\ell} \frac{(-1)^{\ell-n}}{(\ell-n) !} \sum_{k=1}^{n} \frac{1}{k ! k^{n-k}}$.

which is formula (27) with $m=0$.

Expected reward of later players. We focus on the reward of the $m+1$-st arrived player, i.e. when $m$ players are already present. We seek now to instantiate formula (10) with $m=n+1$. Let

$x_{m+1}=x\left(t_{m+1}\right), \quad \theta_{m+1}=\frac{x_{m+1}^{\alpha}}{\alpha a} \quad$ and $\quad \sigma_{m+1}^{\ell}=\sigma_{\ell}-\sigma_{m}$.

We easily obtain

$L_{m+n}=b\left[\left[\theta_{m+1}+\sigma_{m+1}^{m+n}-m\left(t-t_{m+1}\right)-(m+n) t\right]_{+}^{p}\right.$.

An analysis completely parallel to the previous one leads to

$M_{m+1}^{m+n}=\frac{b}{P_{p}(n)} \sum_{k=1}^{n} \frac{(-1)^{k-1}}{(n-k) !(k-1) !} \llbracket \theta_{m+1}-(m+k)\left(t-t_{m+1}\right) \rrbracket_{+}^{p+n-1}$.

Using the similarity with formula (A.3), we get formula (27):

$V_{m+1}=x_{m+1} \sum_{\ell=1}^{\infty} \frac{q^{\ell-1}}{P_{p+1}(\ell)} \sum_{n=1}^{\ell} \frac{(-1)^{\ell-n}}{(\ell-n) !} \sum_{k=1}^{n} \frac{1}{k !(m+k)^{n-k}}$.

as desired.

\section{Appendix B. Simple sharing and the evolution of fungal plant parasites}

The "Simple sharing" example may fit biotrophic fungal plant parasites such as cereal rusts. These fungi travel as airborne spores. When falling on a plant, a spore may germinate and the fungus may penetrate the plant tissue. Such an infection results in a very small (say $1 \mathrm{~mm}^{2}$ large) lesion. After a latent period during which the fungus takes up the products of the plant host's photosynthesis, the lesion starts releasing a new generation of spores. 
There may be hundreds of lesions (foragers) on the same plant (patch). Each lesion was created following the random arrival of a spore, which may be modeled as a Poisson event of intensity $\lambda$. The lesions may remain active until host death (which may be modeled as a Poisson event of intensity $\delta$ ) or the end of the season $(t=T)$. In other words, it is reasonable to assume that no lesion (forager) stops exploiting the plant (patch) before the others. Under this assumption, the individual uptake rate of the resource cannot increase.

Besides, the number of spores produced per unit time per lesion has been shown to reach a plateau after a sufficient number of lesions occur on the same leaf in e.g. Puccinia graminis, the wheat stem rust fungus (see [21, Fig. 2]). This indicates that there is a maximum resource flow that can be extracted from the host and shared among the lesions. This is likely due to the fact that lesions share the same flow of photosynthates and other nutrients provided daily by the plant. Hence the fit with the "Simple sharing" example.

The latent period may be genetically determined, as in e.g. Puccinia triticina, the fungal pathogen causing leaf rust on wheat [30]. Moreover, there is evidence for a positive correlation between the latent period and the number of spores produced per unit time per unit area of sporulating tissue in e.g. P. triticina [22] (see also [14] for a similar trade-off in a different species). Such a trade-off is explained by the fact that shorter latent periods provide less time for the fungus to take up resources from the host, which results in the production of fewer spores [30].

Since the latent period corresponds to a period during which the parasite takes up the host's resources before it can travel to a new host, it is analogous to the patch residence time in foraging theory. In addition, host resources diverted by the fungus are converted into spores (offspring) i.e. into fitness. Consequently, one may wonder what is the optimal latent period given that the longer the latent period, the greater the fitness accumulated prior to departure and infection of a new host.

Let $\theta$ be the mean travel time of a spore. Let $\mathcal{L}$ be the latent period. Following the above analogy with optimal foraging theory, we define an optimal (or evolutionarily stable) latent period as maximizing the following fitness measure (per unit time):

$F(\mathcal{L})=\frac{W(\mathcal{L})}{\theta+\mathcal{L}}$

where $W$ is the fitness accumulated by a single lesion averaged over all possible arrival ranks:

$W(\mathcal{L})=\mathbb{E}_{m} V_{m}(\mathcal{L})$

with e.g., for $m=1$,

$V_{1}(\mathcal{L})=a \int_{0}^{\mathcal{L}} \mathrm{e}^{-\delta t} \frac{1-\mathrm{e}^{-\lambda t}}{\lambda t} \mathrm{~d} t$.

after Theorem 3. More specifically, letting $M=m(T)$ being the total number of lesions on the patch at the end of the season (a Poisson-distributed random variable), we may write:

$W(\mathcal{L})=\mathbb{E}_{M}\left[\frac{1}{M} \sum_{m=1}^{M} V_{m}(\mathcal{L})\right]$,

assuming that each rank of arrival is equiprobable (short latent periods confer no advantage in terms of arrival rank), with probability $1 / M$. Hence,

$W(\mathcal{L})=\sum_{M=1}^{\infty} \mathrm{e}^{-\lambda T} \frac{(\lambda T)^{M}}{M !}\left[\frac{1}{M} \sum_{m=1}^{M} V_{m}(\mathcal{L})\right]$,

Performing the maximization using one of the formulas for $V_{m}(\mathcal{L})$ is left for a future paper, but it can be conjectured that $W(\mathcal{L})$ is increasing, concave and differentiable for all $\mathcal{L}$ so that a classical optimal foraging argument holds: there is a unique $\mathcal{L}^{\star}$ which corresponds to the point where the tangent of $W(\mathcal{L})$ passes through the point $(-\theta, 0)$. Hence the greater the mean travel time of a spore, the greater the optimal latent period.

An open question left is whether increasing $\lambda$ (higher infections frequency) results in decreasing the optimal latent period, as can be expected.

\section{References}

[1] P. Bernhard, F. Hamelin, Simple signaling games of sexual selection (Grafen's revisited), J. Math. Biol. 69 (2014) 1719-1742.

[2] E.-K. Boukas, A. Haurie, Manufacturing flow control and preventive maintenance: a stochastic control approach, IEEE Trans. Autom. Control 35 (1990) 1024-1031.

[3] E. Charnov, Optimal foraging: the marginal value theorem, Theor. Popul. Biol. 9 (1976) 129-136.

[4] C.W. Clark, M. Mangel, The evolutionary advantages of group foraging, Theor. Popul. Biol. 30 (1986) 45-75.

[5] M.H.A. Davis, Piecewise-deterministic markov processes: A general class of non-diffusion stochastic models, J. R. Stat. Soc. B 46 (1984) 355-388.

[6] M.H.A. Davis, Control of piecewise-deterministic processes via discrete-time dynamic programming, in: Stochastic Differential Systems, in: Lecture Notes in Control and Information Sciences, vol. 78, Springer, 1985, pp. 140-150.

[7] S.D. Fretwell, H.L. Lucas, On territorial behavior and other factors influencing habitat distribution in birds. i. theoretical development, Acta Biotheor. 19 (1969) 16-36.

[8] L.-A. Giraldeau, T. Caraco, Social foraging theory, in: Monographs in Behavior and Ecology, Princeton University Press, 2000.

[9] J. Haigh, C. Cannings, The n-person war of attrition, Acta Appl. Math. 14 (1989) 59-74.

[10] F. Hamelin, P. Bernhard, P. Nain, É. Wajnberg, Foraging under competition: evolutionarily stable patch-leaving strategies with random arrival times. 1. scramble competition, in: T. Vincent (Ed.), Advances in Dynamic Game Theory, Annals of the International Society of Dynamic Games, vol. 9, Birkhaüser, 2007a, pp. $327-348$.

[11] F. Hamelin, P. Bernhard, A.J. Shaiju, É. Wajnberg, Diet selection as a differential foraging game, SIAM J. Control Optim. 46 (2007b) 1539-1561.

[12] F. Hamelin, P. Bernhard, A.J. Shaiju, É. Wajnberg, Foraging under competition: evolutionarily stable patch-leaving strategies with random arrival times. 2 interference competition, in: T. Vincent (Ed.), Advances in Dynamic Game Theory, Annals of the International Society of Dynamic Games, vol. 9, Birkhaüser, 2007c, pp. 349-365.

[13] A. Haurie, A. Leizarowitz, Ch. van Delft, Boundedly optimal control of piecewise deterministic systems, Eur. J. Oper. Res. 73 (1994) 237-251.

[14] V. Héraudet, L. Salvaudon, J.A. Shykoff, Trade-off between latent period and transmission success of a plant pathogen revealed by phenotypic correlations, Evolut. Ecol. Res. 10 (6) (2008) 913-924.

[15] C.S. Holling, The components of predation as revealed by a study of smallmammal predation of the european pine sawfly, Can. Entomol. 91 (1959) 293320.

[16] A. Kacelnik, J.R. Krebs, C. Bernstein, The ideal free distribution and predatorprey populations, Trends Ecol. Evolut. 7 (1992) 50-55.

[17] R.H. MacArthur, E.R. Pianka, On the optimal use of a patchy environment, Am. Nat. 100 (1966) 603-609.

[18] J.M. Smith, Evolution and the Theory of Games, Cambridge University Press, Cambridge, U.K., 1982.

[19] J.M. Smith, G.R. Price, The logic of animal conflict, Nature 246 (1973) 15-18.

[20] D. Mollison, Dependence of epidemic and population velocities on basic parameters, Math. Biosci. 107 (1991) 255-287.

[21] M.R. Newton, L.L. Kinkel, K.J. Leonard, Competition and density-dependent fitness in a plant parasitic fungus, Ecology 78 (6) (1997) 1774-1784.

[22] B. Pariaud, F. van den Berg, F. van den Bosch, S.J. Powers, O. Kaltz, C. Lannou, Shared influence of pathogen and host genetics on a trade-off between latent period and spore production capacity in the wheat pathogen, puccinia triticina, Evolut. Appl. 6 (2) (2013) 303-312.

[23] J. Pastor, Mathematical Ecology, Wiley-Blackwell, 2008.

[24] R. Rishel, Dynamic programming and minimum principles for systems with jump markov disturbances, SIAM J. Control 13 (1975) 338-371.

[25] G.D. Ruxton, C. Fraser, M. Broom, An evolutionarily stable joining policy for group foragers, Behav. Ecol. 16 (2005) 856-864.

[26] D.W. Stephens, J.S. Brown, R.C. Ydenberg, Foraging: Behaviour and Ecology, University of Chicago Press, 2008.

[27] D.D. Sworder, Feedback control of a class of linear systems with jump parameters, IEEE Trans. Autom. Control AC-14 (1969) 9-14.

[28] P.D. Taylor, L.B. Jonker, Evolutionarily stable strategies and game dynamics, Math. Biosci. 40 (1978) 145-156.

[29] H. Tembine, Evolutionary networking games, in: Game Theory for Wireless Communications and Networking, Auerbach Publications, CRC Press, 2010, pp. 133-158.

[30] F. Van den Berg, S. Gaucel, C. Lannou, C.A. Gilligan, F. van den Bosch, High levels of auto-infection in plant pathogens favour short latent periods: a theoretical approach, Evolut. Ecol. 27 (2) (2013) 409-428.

[31] D. Vermes, Optimal control of piecewise deterministic markov process, Stochastics 14 (1985) 165-207. 